\title{
Liderazgo complejo como elemento para mejorar el índice de aprobación
}

\section{Complex leadership as element to improve the approval rate}

TOVAR-ROSAS, Claudia Rocío†*, GARZA-MOYA, Luis Roberto, ARREOLA-BURCIAGA, Josué Mizraim y RODRÍGUEZ-ALANÍS, Francisco de Borja

Universidad Politécnica de Gómez Palacio, México.

ID $1^{\text {er }}$ Autor: Claudia Rocío, Tovar-Rosas / ORC ID: 0000-0002-8238-7493, CVU CONACYT ID: 745074

ID $1^{\text {er }}$ Coautor: Luis Roberto, Garza-Moya / ORC ID: 0000-0002-5740-1476, CVU CONACYT ID: 68866

ID $2^{\text {do }}$ Coautor: Josué Mizraim, Arreola-Burciaga / ORC ID: 0000-0002-2722-1386, CVU CONACYT ID: 769055

ID $3^{\text {er }}$ Coautor: Francisco de Borja, Rodríguez-Alanís / ORC ID: 0000-0002-2949-1785, CVU CONACYT ID: 719064

DOI: $10.35429 / J C P .2020 .11 .4 .1 .8$

Recibido 10 de Enero, 2020; Aceptado 30 de Junio, 2020

Resumen

Actualmente vivimos en un mundo globalizado, el cual exige tener ciertos conocimientos y habilidades para poder desarrollar las actividades laborales diarias, tales como el conocimiento de algún lenguaje de programación, bases de datos, entre otros. Muchas de las empresas actualmente solicitan a las universidades que los egresados no tengan únicamente los conocimientos necesarios para desarrollar las actividades, si no que buscan que los titulados cuenten con habilidades del ser, para desplegar sus conocimientos tal como es, el trabajo en equipo, el liderazgo, la elaboración de cronogramas de trabajo, entre otros. Por todo lo anterior se buscó una forma de impartir en el aula no solo los conocimientos esenciales de una materia, sino que se investigó una forma de explotar las habilidades propias de cada estudiante dentro del aula, es por ello que se implementó el liderazgo complejo en la Universidad Politécnica de Gómez Palacio, específicamente en el grado de 6to. Cuatrimestre de la carrera de Tecnologías de la Información, siendo implementado en la materia de base de datos, con ello se buscó una estrategia para mejorar el índice de aprobación, ya que está materia en específico es un tema difícil de entender para los alumnos de la carrera de TI.

Liderazgo complejo, Índice de aprobación, Estrategias de aprendizaje

\begin{abstract}
We currently live in a globalized world, which requires having certain knowledge and skills in order to carry out daily work activities, such as knowledge of some programming language, databases, among others. Many of the companies currently request universities that graduates not only have the necessary knowledge to carry out activities, but rather that graduates have the skills of being, to display their knowledge as it is, teamwork, leadership, the development of work schedules, among others. For all of the above, a way was sought to impart not only the essential knowledge of a subject in the classroom, but also a way to exploit the abilities of each student within the classroom, which is why complex leadership was implemented. at the "Universidad Politécnica of Gómez Palacio", specifically in the 6th quarter grade of the Information Technology degree, implemented in the database subject, with this, a strategy was sought to improve the approval rate, since this specific subject is a difficult subject for the students of the degree to understand.
\end{abstract}

Citación: TOVAR-ROSAS, Claudia Rocío, GARZA-MOYA, Luis Roberto, ARREOLA-BURCIAGA, Josué Mizraim y RODRÍGUEZ-ALANÍS, Francisco de Borja. Liderazgo complejo como elemento para mejorar el índice de aprobación. Revista de Pedagogía Crítica. 2020. 4-11:1-8.

\footnotetext{
* Correspondencia al Autor (Correo electrónico: ctovar@upgop.edu.mx)

$\uparrow$ Investigador contribuyendo como primer autor.
} 


\section{Introducción}

En las aulas escolares podemos encontrar estudiantes con diferentes habilidades destacables, las cuales aportaran en un futuro sus actividades en el campo laboral, estos alumnos enriquecen el aula puesto que al estar en conjunto se desarrolla de diferente forma la actividad enseñanza - aprendizaje, además de superar las expectativas del curso al estar presentes dentro de las diversas asignaturas.

A pesar de que se tienen alumnos con diversas habilidades en las aulas, nos centraremos en una actividad fundamental para la mayoría de las empresas actuales, la cual consiste en el liderazgo complejo, ya que tal como indica. (Gil, Alcover, Rico, \& SánchezManzanares, 2011), este enfoque aplicado al liderazgo identifica tres ejes de complejidad: cognitiva, social y conductual, de forma que el líder debe ser capaz de abordar los acontecimientos desde distintas perspectivas, desempeñar distintos roles y ejecutar distintas conductas en función de las demandas de la situación, tal como lo indica el autor, los líderes de proyectos y jefes de producción, así como emprendedores deben de tener una visión amplia sobre las actividades a realizar, ya que no se deben de enfocar en una actividad continua y rutinaria, si no que deben aportar a los departamentos que afectan directa e indirectamente el producto que realizan.

Actualmente la mayoría de los empresas solicitan que los egresados de las instituciones de educación superior (IES) tengan la habilidad de ser líderes, trabajar en equipo y manejar diversas actividades tanto dentro como fuera de su área de conocimiento, tal como indica (Gil et al., 2011) cuando se aproxima el acceso al mundo del trabajo, los individuos intentan identificar el tipo de trabajo que les interesa considerando sus intereses, valores y preferencias laborales, solicitando información sobre puestos de trabajo, profesiones y empleos de conocidos, amigos, familiares y centrándose posteriormente en la formación o el aprendizaje necesarios, es por ello que las universidades suelen realizar diversas evaluaciones y evidencias que consisten en actividades para desarrollar los conocimientos.
Así como el afianzamiento de las diversas habilidades, una de ellas, la cual toma mayor presencia dentro de las aulas escolares al realizar un proyecto, exposición, trabajo colaborativo, entre otros, es el liderazgo que se tiene al realizar dichas actividades tanto dentro del equipo de trabajo con más de un compañero de actividades o a nivel grupal.

Algunas de las actividades realizadas en el aula están enlazadas es decir son incrementales, es por ello que la mayoría de esos proyectos son desarrollados en forma colaborativa, en equipos de trabajo, los cuales crean un producto a partir de las instrucciones o necesidades aportadas por el docente, el cual funge como "el cliente" y dictamina una serie de necesidades específicas a desarrollar dentro del proyecto asignado, así como las diversas características de los requerimientos solicitados para validar el producto final; los grupos suelen dividir el trabajo en forma de islas de desarrollo apartadas unas de otras, las cuales entregan un producto final pero creado de manera simple, ya que estas islas de trabajo suelen ser integradas por pocos estudiantes, por lo tanto el tiempo se ve reducido al tener que analizar, diseñar, desarrollar y probar el producto creado, por un equipo de trabajo mínimo y por ende la entrega del proyecto final puede ser diferente a lo esperado por los desarrolladores, es por ello que al considerar elaborar un proyecto enfocado a resolver un problema se consideró el modificar la estrategia grupal con el fin de minimizar el trabajo y maximizar la calidad del mismo.

Uno de los indicadores que tiene mayor influencia en el estudio del rendimiento institucional de cualquier asignatura impartida hacia cierto público meta, es el de rendimiento escolar, el cual tal como lo indica la (SEP, 2019), este indicador se obtiene de dividir el número total de alumnos aprobados entre el total de alumnos existentes en un grado, al final del ciclo escolar, es importante que el indicador se maneje en un número mayor a la cantidad de alumnos reprobados, puesto que en caso contrario se deben analizar los motivos de la alta reprobación, así como las diversas estrategias a implementar, las cuales deben de ser indicadas por el docente a cargo y supervisadas por el coordinador de la carrera. 
Por ello es necesario tomar dicho indicador como base para modificar las técnicas de enseñanza impartidas en el aula y con ello, promover el conocimiento y el desarrollo de las habilidades para el aumento de la eficiencia terminal en las asignaturas con un mayor índice de deserción.

Las materias con mayor incidencia en reprobación y deserción dentro de la carrera de Ingeniería enTecnologías de la Información (ITI) en la Universidad Politécnica de Gómez Palacio (UPGOP), son las que guardan una estrecha relación con el análisis y manejo de la información, es por ello que en el presente artículo abarcaremos una de las asignaturas con un alto porcentaje de reprobación dentro de la institución, la materia de bases de datos, la cual consta de 4 unidades divididas a su vez en entregas de evidencias en su mayoría en forma mensual, estas consisten en proporcionar pequeñas distribuciones de un proyecto final, el cual esta formulado por el compendio de todas las unidades en un solo producto, las recomendaciones en el manual de trabajo es que sean desarrolladas en islas de trabajo y su evaluación posterior sea con una entrega final en formato digital.

A partir de lo anterior se propuso un cambio en cuanto a la técnica propuesta para la dirección de las actividades dentro del aula, ya que los indicadores de eficiencia terminal aplicados a la asignatura indicaban que el uso de la técnica actual de desarrollo de proyectos, así como la comprensión de los diversos temas vistos dentro de la asignatura no eran comprendidos por los alumnos, por ende ellos tendían a reprobar varias veces la misma materia, además cabe destacar que el trabajo realizado dentro del aula se reparte de forma equitativa y de acuerdo a las habilidades mostradas en las diversas materias de la carrera. En el desarrollo del presente artículo se analiza las características grupales, así como algunas de las deficiencias que tienen los estudiantes al momento de ingresar a materias de análisis como lo es bases de datos, además de proporcionar algunas de las peculiaridades estructurales de la institución donde se realizó el presente proyecto, tales como el empleo de los diversos laboratorios de cómputo en los cuales se desarrollan los proyectos, así como las diversas metodologías de enseñanza - aprendizaje implementadas por el docente.
La metodología por desarrollar nos aportó un punto a seguir, ya que se analizaron los resultados provenientes de las últimas 4 generaciones anteriores correspondientes a la misma materia, así como las estrategias implementadas en esos momentos, además de la evolución de ellas y sus resultados.

Como paso final se muestran los resultados obtenidos en comparación a las otras generaciones analizadas, además de la estrategia implementada y las conclusiones que llevaron a la culminación del presente artículo.

\section{Desarrollo}

La materia de base de datos fue impartida en el cuatrimestre Mayo - Agosto, correspondiente al sexto ciclo de formación de la carrera de ITI, actualmente está carrera cuenta con más de 10 generaciones de egresados, los cuales cursaron en algún momento de su especialidad la materia de base de datos.

Como parte de la actualización de la currícula escolar realizada por la CGUTyP, se llevaron a cabo algunas modificaciones, entre ellas la reubicación de la asignatura de bases de datos en el período de Enero - Abril, correspondiente al quinto cuatrimestre, implementandose a partir el año 2018.

Algunas de las deficiencias detectadas en los alumnos antes de ingresar a la materia de base de datos fueron:

Falta de lógica en cuestiones simples y rutinarias.

- No relacionar la utilidad de enlazar materias, es decir implementar conocimientos pasados en las materias actuales.

Poco conocimiento de la utilidad de algunas herramientas proporcionadas en clase.

Es por ello que, al analizar dicha situación, esto representó un punto de partida al momento de hacer la estrategia de evaluación para los alumnos que cursarían la materia de base de datos en Enero. 
Las características presentes en la institución son:

Actualmente cuenta con cuatro ingenierías y una licenciatura.

Cuenta con nueve centros de cómputo para la instrucción de las diversas materias impartidas por las especialidades.

Se tiene una matrícula de más de 1,300 alumnos inscritos.

Tres de las carreras se encuentran acreditadas por CACEI y COPAES, en espera de la acreditación de las otras dos carreras.

\section{Metodología por desarrollar}

Como primer paso se procedió a examinar las materias con un mayor índice de reprobación a lo largo de cuatro generaciones anteriores a la actual, analizando las materias con mayor incidencia en grandes índices de reprobación, teniendo como principal tendencia la materia de bases de datos.

Para el segundo paso se presentaron los hallazgos en la academia de ITI, con lo cual se propusieron diversas estrategias de aprendizaje, desde las centradas en el docente, hasta las centradas en la comunidad.

Como paso siguiente se procedió a revisar las características presentadas en el grupo, desde sus habilidades desarrolladas hasta este punto de formación, así como las presentadas por cada alumno.

Como conclusión del paso anterior se procedió a elegir el modelo de liderazgo complejo, ya que se analizaron varias de sus características, las cuales se acoplan a la perfección con la forma de trabajar de los grupos en los cuales se implementaría, además de considerar la forma de realizar la rúbrica implementando algunos de los componentes vistos en el artículo Evaluación del liderazgo organizacional y directivo en las escuelas de medicina de México por Olivares et al., (2016), empleándolo así como un apoyo para la realización de la rúbrica pertinente.
Al tener considerado lo anterior se procedió a implementar dentro del aula de clases, siendo desarrollado en dos grupos uno con 18 alumnos y el otro con 28 , ambos grupos tenían acceso a los centros de cómputo en un horario establecido, además de poder usarlos de forma libre a contra turno, ambos grupos son del turno matutino y su currícula corresponde a la 2018.

Como siguiente paso se procedió a informar sobre las rúbricas y mecanismos de evaluación (Tabla 1), la forma de desarrollar las actividades, así como la designación de los roles en los equipos de trabajo.

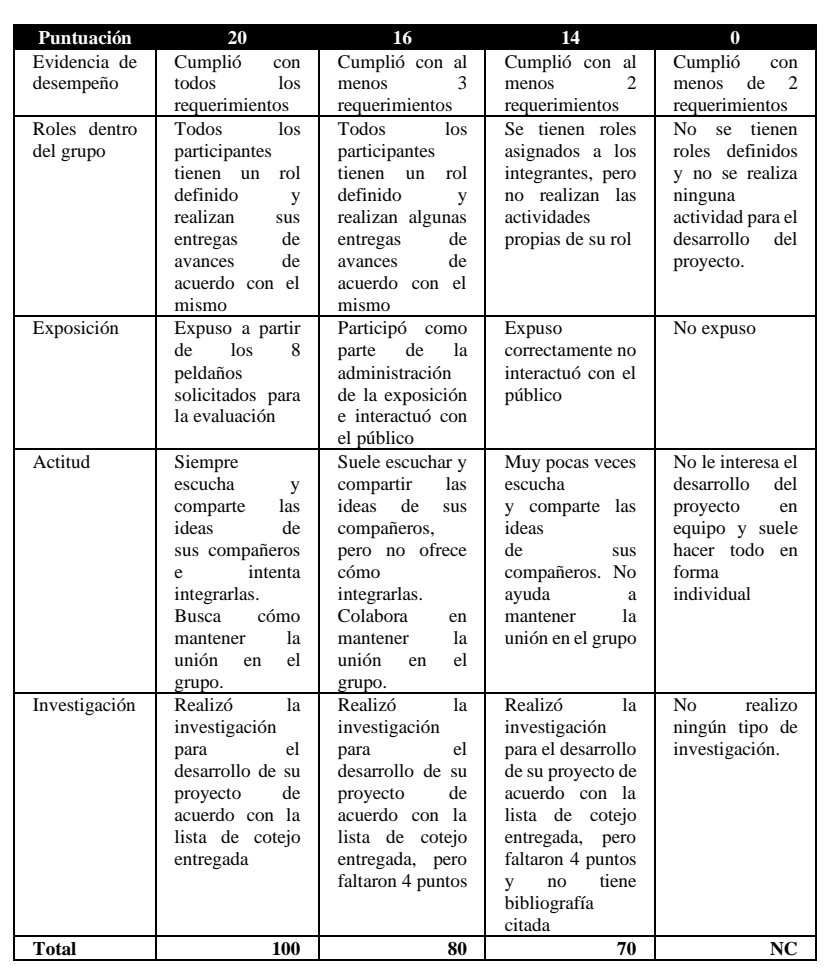

Tabla 1 Rúbrica de evaluación

Fuente: Academia de ITI

La forma de desarrollar los equipos fue la siguiente:

- Realizar equipos de trabajo colaborativo de una cantidad menor a seis alumnos por equipo y mayor a tres.

- Entre los integrantes se debería de proponer a un administrador, el cual estará en contacto con los demás administradores y a su vez con el líder de proyecto.

- Los integrantes tendrán los puestos de analistas, diseñadores y programadores dentro de su equipo de trabajo. 
- El proyecto por desarrollar constará de varias etapas, las cuales son incrementales y serán revisadas unidad tras unidad, se debe de tener contacto con todos los equipos de trabajo, ya que el proyecto será uno solo para ser desarrollado por el grupo en su totalidad.

- $\quad$ Entre los integrantes del equipo se deberá tener un tester, el cual revise los avances y proponga cambios en caso de detectar anomalías en el sistema.

Cada equipo contará con un módulo a desarrollar, al finalizar cada etapa el líder de equipo debe de presentar dichos avances a una junta de expertos "clientes", los cuales validarán los avances.

En cada término de una etapa del modelo de desarrollo seleccionado, se debe exponer los avances del proyecto, al finalizar será presentado a clientes potenciales, los cuales serán invitados para conocer los proyectos desarrollados dentro de la institución.

Al implementar el siguiente paso se tomó en consideración lo indicado por (Cornejo, 2014), en un primer momento se analiza el encargo desarrollado por los actores organizacionales, poniendo posiblemente en tensión los discursos de acuerdo a la jerarquía, rol, problemática, interpretación que realizan, etc, es decir la evaluación dependerá de las actividades a realizar por los equipos de trabajo, dividiendo las funciones de acuerdo a sus perfiles y habilidades, además de las necesidades detectadas por parte del administrador del equipo entorno al proyecto a realizar, junto con los requerimientos del mismo.

Para la revisión del cumplimiento de los pasos anteriores se procedió a emplear una rúbrica de evaluación, ya que se debe analizar el trabajo en equipo, tal como indica (Chica, 2011), es una de las competencias a las que se hace más referencia en diferentes ámbitos, tanto académico, profesional como social. Incluso en documentos de ámbito nacional e internacional, se reconoce la importancia de esta capacidad de forma explícita, es por ello por lo que es relevante la forma de evaluación, puesto que los involucrados deberán cumplir con su rol dentro del trabajo.
Además de considerar la evaluación de los líderes que llevaran a cabo la recopilación de la información, así como la revisión continua de las variables y la constante retroalimentación por parte del tester y la presentación de la información hacia el líder de equipo, el cual cuenta con un cronograma de trabajo muy reducido, por lo cual dichos líderes deberían de tener ciertas características, tal como indica (Lupano Perugini \& Castro Solano, 2006), los líderes se preocupan por las necesidades de cada una de las personas que componen su equipo o división y además las hacen participar en la toma de decisiones, es por ello que se debe de tener un líder competente, el cual sea activo dentro de la toma de decisiones que se tendrán dentro del proyecto a realizar.

Como siguiente paso se realizaron las actividades propias de las unidades designando diferentes roles a los estudiantes y visualizándolos en un proyecto del mundo real, tal como lo fue el emplear una base de datos para cada grupo, tomando en consideración el planteamiento del problema, para el primer grupo, el cual está conformado por 18 alumnos, se otorgó el proyecto a realizar para una tienda departamental, tomando en cuenta todos sus módulos y el nivel de actividades diarias, además de considerar el modelado de la base de datos, la seguridad a implementar e incluso el gestor de base de datos a utilizar, para el segundo grupo se propuso elaborar un proyecto a implementar en un aeropuerto, desde la compra de boletos, hasta el manejo del personal y el control de los vuelos.

Una vez terminado el paso anterior se procedió a dar retroalimentación unidad tras unidad para el cumplimiento de los requisitos por parte del cliente, además de evaluar la interacción de los asociados tal como indica (Pease Dreibelbis, 2011), conviene integrar la lógica de la técnica del rompecabezas a las actividades programadas, donde cada individuo tiene que trabajar una "pieza" y garantizar que los demás la comprendan, para luego sumar entre todos las diversas piezas, y dar origen a un producto final nuevo y de mayor complejidad, es por ello que se debe de tener en cuenta la elaboración de una función modular, desde el equipo de trabajo hacia los demás equipos. 
Al término de los proyectos se solicitó a los líderes de equipo exponer frente a un grupo de personas interesadas en el desarrollo de dichos productos, por lo cual se debía grabar un clip de video, además de presentar el proyecto desde su primera composición hasta su terminación y la utilidad que presentaría el ser implementado en las diversas empresas interesadas.

\section{Resultados}

Algunos de los resultados obtenidos a partir de la implementación de los proyectos fue la elaboración de avances incrementales desarrollados en base a las diversas fundamentos otorgados por parte del instructor (Figura 1).

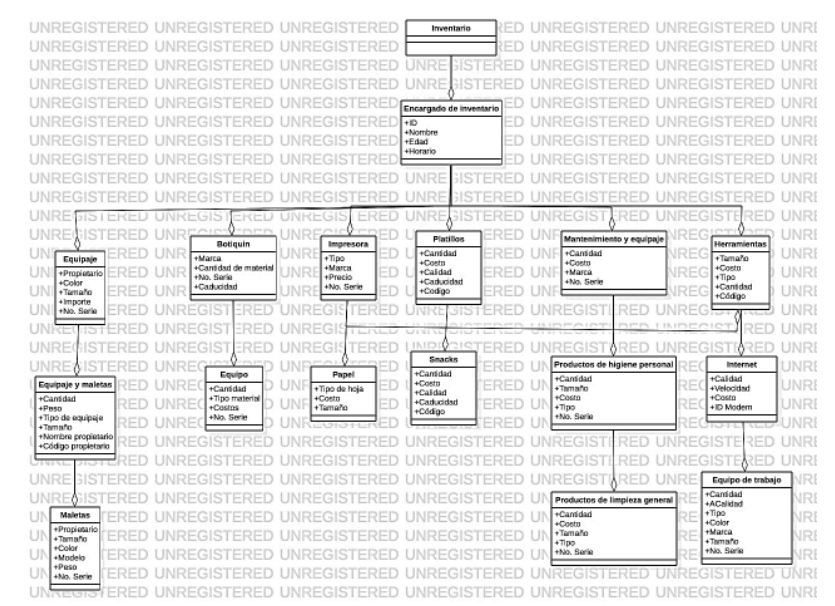

Figura 1 Modelo Entidad - Relación

Fuente: Estudiantes de quinto cuatrimestre de la carrera de ITI

A partir de dichos modelos se logró cambiar los diagramas realizados a mano e implementar por medio de Software algunas modificaciones para que fuese entendible el proyecto hacia el cliente a partir de la implementación de algunos temas vistos en clases (Figura 2)

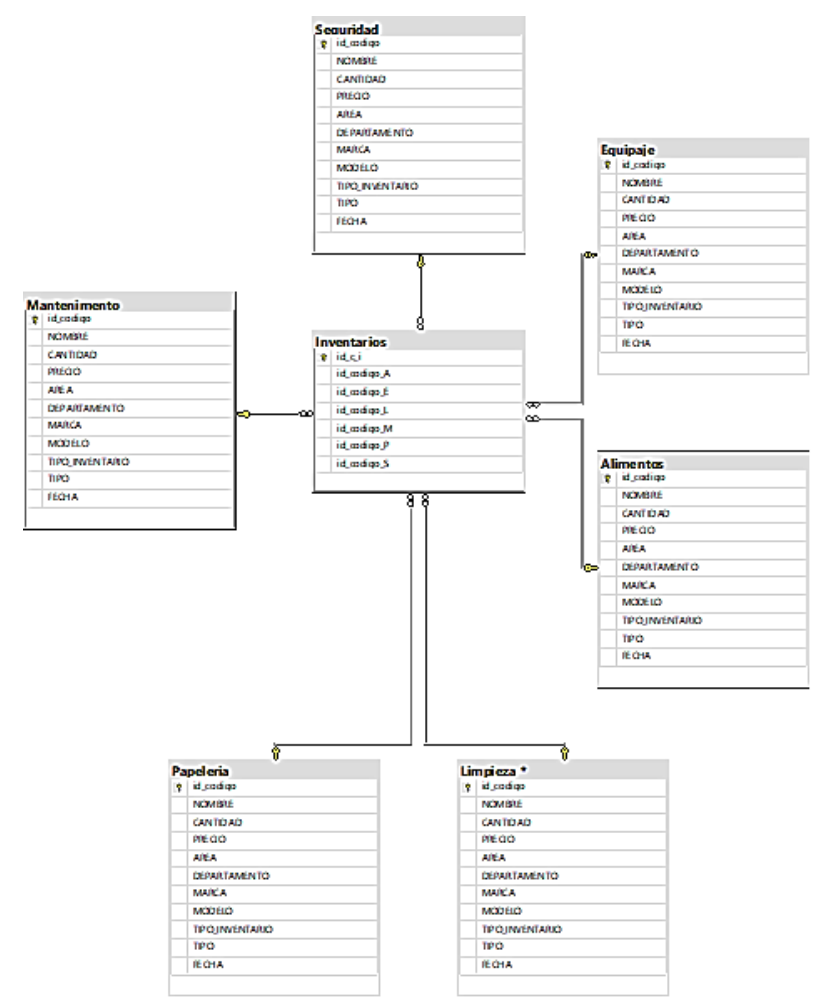

Figura 2 Modelado de la base de datos en SQL Server Fuente: Estudiantes de quinto cuatrimestre de la carrera de ITI

Como primer interés al desarrollar el presente modelo, es el de comprender las bases de datos, tanto desde el diagramado, hasta la implementación de subconsultas, las cuales apoyan a desplegar de forma filtrada la información almacenada.

Los resultados obtenidos a partir del empleo del modelo de liderazgo complejo fueron diferente al esperado, ya que el índice de aprobación rebasó las expectativas deseadas, puesto que se tenía como base el subir un $15 \%$ el indicador, mientras que el resultado fue elevado a un $42 \%$ con respecto al índice del año anterior (Tabla 2).

\begin{tabular}{|l|r|r|r|r|r|r|}
\hline $\begin{array}{l}\text { Total } \\
\text { Ingreso }\end{array}$ & $\mathbf{2 0 1 5}$ & $\mathbf{2 0 1 6}$ & $\mathbf{2 0 1 7}$ & $\mathbf{2 0 1 8}$ & $\mathbf{2 0 1 9}$ & $\mathbf{2 0 2 0}$ \\
\hline Aprobados & 16 & 53 & 39 & 64 & 53 & 46 \\
\hline Reprobados & 16 & 32 & 23 & 12 & 24 & 2 \\
\hline $\begin{array}{l}\text { Índice } \\
\text { Aprobación }\end{array}$ & $50 \%$ & $40 \%$ & $41 \%$ & $81 \%$ & $54 \%$ & $96 \%$ \\
\hline Rezagados & 1 & 4 & 3 & 2 & 1 & 0 \\
\hline Promedio & 3.68 & 3.31 & 3.31 & 6.90 & 4.38 & 8.27 \\
\hline
\end{tabular}

Tabla 2 Indicadores 6 generaciones

Fuente: Departamento de estadísticas UPGOP 
Tal como se muestra en la (Tabla 1), a lo largo de las generaciones se han tenido diferentes comportamientos correspondientes a la materia de base de datos, ya que además de contar con generaciones numerosas, estas no han influido en cuanto a los estadísticos de índice de aprobación, puesto que estos números indican que a partir de la metodología empleada se ve un incremento exponencial en cuanto a generaciones anteriores, además del aumento en el promedio grupal tomando en consideración la cantidad de alumnos, índices de reprobación y rezago educativo presentado a partir de las generaciones analizadas.

Como se observa en la tabla en la generación 2018 se implementó el trabajo modular, pero no tan estructurado como se presentó en este artículo, mientras que en las generaciones se empleó la evaluación por islas de trabajo individuales, lo cual llevo a que los alumnos tuviesen un bajo desempeño, por lo cual se implementó la realización de proyectos grupales, en la generación 2019 se tiene un comportamiento errático, ya que para comprobar la hipótesis se procedió a continuar con proyectos elaborados con islas individuales, lo que trajo consigo el replanteamiento del trabajo en equipo pero empleando el modelo de liderazgo complejo, por lo cual los resultados mostrados en el 2020 (Gráfico 1), demuestran un aumento considerable en cuanto a la aplicación del modelo de islas, ya que el trabajo colaborativo y guiado por líderes de equipo demostró que es la herramienta a implementar continuamente para el desarrollo de los proyectos posteriores, además de ver una tendencia positiva.

\section{Índice de aprobación materia base de} datos

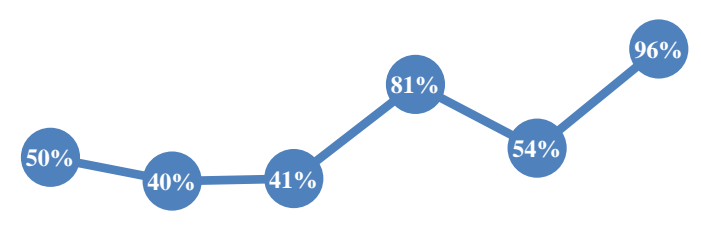

2015-2 2016-2 2017-2 2018-2 2019-2 2020-1

\section{Agradecimiento}

Se tiene un agradecimiento a la UPGOP, ya que está institución otorgó las materias primas para el desarrollo del proyecto, además de agradecer a los alumnos de quinto cuatrimestre por llevar a cabo el modelo en tiempo y forma, además de entregar un producto profesional y de calidad, además agradecer a la academia de ITI, ya que entre todos se logró llegar a un modelo conveniente para la institución y los alumnos a partir de la colaboración tenida en las reuniones.

\section{Conclusiones}

A partir de los hallazgos encontrados pudimos deducir que el empleo del presente proyecto fue un punto positivo, ya que se logró aumentar el índice de aprobación, además con la implementación de este se logró conectar un modelo de trabajo eficiente con las estrategias de aprendizaje, además de desarrollar las habilidades innatas de los estudiantes.

Uno de los puntos a recalcar es la relevancia de buscar estrategias de aprendizaje surgen a partir de las nuevas tendencias y necesidades detectadas por grupo, además de las dinámicas grupales que se manejan en cualquier IES.

Otra de las áreas de oportunidad que pudieron ser reforzadas a través del proyecto realizado fue el seguir un proceso de mayor proyección contribuyendo al plan de mejora de la acreditación CACEI, ya que algunas de las observaciones por dicha acreditación es el buscar la mejora continua educativa sin perder de vista el perfil educativo, así como la aportación de nuevas herramientas y habilidades para la productividad del alumno al egresar.

Como parte del trabajo a futuro será la implementación del modelo de liderazgo complejo en materias que representen retos para los alumnos de la carrera de ITI en primera instancia y después la reproducción del mismo en otras especialidades que se tienen en la institución.
Gráfico 1 Incremento de índice de aprobación Fuente: Departamento de estadísticas UPGOP 


\section{Referencias}

Chica, E., \& Resumen, M. (2011). una propuesta de evaluación para el trabaJo en Grupo mediante rÚbrica TITLE: A PROPOSAL FOR EVALUATION GROUP WORK BY HEADING. Escuela Abierta, 14, 67-81. Retrieved from http://www.ceuandalucia.es/escuelaabierta/pdf/ articulos_ea14pdf/ea14_chica.pdf

Cornejo, H. (2014). Liderazgo Complejo En Procesos De Implementación-Apropiación Tecnológica. 5. Retrieved from https://www.aacademica.org/000-035/332

Gil, F., Alcover, C. M., Rico, R., \& SánchezManzanares, M. (2011). Nuevas formas en equipos de trabajo. Papeles Del Psicólogo, Vol.32(No.1), 38-47. Retrieved from https://dialnet.unirioja.es/servlet/articulo?codig $\mathrm{o}=3406744 \% 0 \mathrm{Ahttp}: / / \mathrm{www} \cdot$ redalyc.org/articulo .oa?id=77817210005

Lupano Perugini, M. L., \& Castro Solano, A. (2006). Estudios sobre el liderazgo. Teorías y evaluación. Psicodebate, 6(0), 107. https://doi.org/10.18682/pd.v6i0.444

Olivares Olivares, Silvia Lizett, Garza Cruz, Alejandra, López Cabrera, Mildred Vanessa, \& Suárez Regalado, Alex Iván. (2016). Evaluación del liderazgo organizacional y directivo en las escuelas de medicina de México. Innovación educativa (México, DF), 16(70), 131-149. Recuperado en 27 de agosto de 2020, de http://www.scielo.org.mx/scielo.php?script=sci _arttext\&pid=S1665-

26732016000100131\&lng=es\&tlng=es.

Pease Dreibelbis, M. A. (2011). Evaluación en trabajo en equipo. $2,1-4$.

SEP. (2019). Lineamientos para la Formulación de Indicadores Educativos. Retrieved from https://www.planeacion.sep.gob.mx/Doc/estadi stica_e_indicadores/lineamientos_formulacion_ de_indicadores.pdf 